\title{
NONLINEAR ERGODIC THEOREMS \\ FOR ASYMPTOTICALLY ALMOST \\ NONEXPANSIVE CURVES IN \\ A HILBERT SPACE
}

\author{
GANG LI AND JONG KYU KIM
}

Received 3 May 2000

We introduce the notion of asymptotically almost nonexpansive curves which include almost-orbits of commutative semigroups of asymptotically nonexpansive type mappings and study the asymptotic behavior and prove nonlinear ergodic theorems for such curves. As applications of our main theorems, we obtain the results on the asymptotic behavior and ergodicity for a commutative semigroup of non-Lipschitzian mappings with nonconvex domains in a Hilbert space.

\section{Introduction}

Let $H$ be a real Hilbert space with norm $\|\cdot\|$ and inner product $(\cdot, \cdot)$. Let $C$ be a nonempty subset of $H$ and $G$ be a commutative semitopological semigroup with identity. In this case, $(G, \succcurlyeq)$ is a directed system when the binary relation " $\succcurlyeq$ " on $G$ is defined by $b \succcurlyeq a$ if and only if there is $c \in G$ such that $a+c=b$. Let $\mathfrak{s}=\{T(t): t \in G\}$ be a semigroup acting on $C$, that is, $T(t+s) x=T(t) T(s) x$ for all $t, s \in G$ and $x \in C$. Recall that a semigroup $\Im$ on $C$ is said to be

(a) nonexpansive if $\|T(t) x-T(t) y\| \leq\|x-y\|$ for $x, y \in C$ and $t \in G$,

(b) asymptotically nonexpansive, [9], if there exists a function $k: G \mapsto[0, \infty)$ with $\lim \sup _{t \in G} k_{t} \leq 1$ such that

$$
\|T(t) x-T(t) y\| \leq k_{t}\|x-y\|
$$

for $x, y \in C$ and $t \in G$,

(c) of asymptotically nonexpansive type, [9], if for each $x \in C$, there is a function $r(\cdot, x): G \mapsto[0, \infty)$ with $\lim _{t \in G} r(t, x)=0$ such that

$$
\|T(t) x-T(t) y\| \leq\|x-y\|+r(t, x) \quad \forall y \in C, t \in G,
$$

where $\lim _{t \in G} \alpha(t)$ denotes the limit of a net $\alpha(\cdot)$ on the directed system $(G, \succcurlyeq)$.

Copyright $\odot 2000$ Hindawi Publishing Corporation

Abstract and Applied Analysis 5:3 (2000) 147-158

2000 Mathematics Subject Classification: 47H09, 47H10, 47H20

URL: http://aaa.hindawi.com/volume-5/S1085337500000312.html 
It is easily seen that $(a) \Rightarrow(b) \Rightarrow(c)$ and that both the inclusions are proper (cf. [9, page 112]).

In 1975, Baillon [1] proved the first nonlinear mean ergodic theorem for nonexpansive mappings in a Hilbert space: let $C$ be a nonempty closed convex subset of a Hilbert space $H$ and let $T$ be a nonexpansive mapping of $C$ into itself. If the set $F(T)$ of fixed points of $T$ is nonempty, then the Cesáro means

$$
S_{n}(x)=\frac{1}{n} \sum_{k=0}^{n-1} T^{k} x
$$

converge weakly as $n \rightarrow \infty$ to a fixed point $y$ of $T$ for each $x \in C$. In this case, letting $y=P x$ for each $x \in C, P$ is a nonexpansive retraction of $C$ onto the fixed point set $F(T)$ of $T$ such that $P T=T P=P$ and $P x \in \overline{\operatorname{conv}}\left\{T^{n} x: n=0,1,2, \ldots\right\}$ for each $x \in C$, where $\overline{\operatorname{conv}} A$ denotes the closure of the convex hull of $A$. The analogous results are given for nonexpansive semigroups by Baillon and Brézis [2] and Brézis and Browder [3]. In [13], Mizoguchi and Takahashi proved a nonlinear ergodic retraction theorem for Lipschitzian semigroups by using the notion of submean.

In this paper, we introduce the notion of asymptotically almost nonexpansive curves which include almost-orbits of commutative semigroups of asymptotically nonexpansive type mappings, and we prove nonlinear ergodic theorems for such curves. As applications of our main theorems, we obtain the results on the asymptotic behavior and ergodicity for a commutative semigroup of non-Lipschitzian mappings with nonconvex domains in a Hilbert space. Our results generalize and improve the previously known results of Baillon [1], Baillon and Brézis [2], Hirano and Takahashi [6], Ishihara and Takahashi [7], Lau, Nishiura, and Takahashi [10], Li and Ma [11, 12], Mizoguchi and Takahashi [13], Takahashi [14, 15], Takahashi and Zhang [16], and Tan and Xu [17] in many directions.

\section{Preliminaries and notations}

Throughout this paper, let $H$ be a real Hilbert space with norm $\|\cdot\|$ and inner product $(\cdot, \cdot)$. Let $G$ be a commutative semitopological semigroup with identity and let $m(G)$ be the Banach space of all bounded real-valued functions on $G$ with the supremum norm. For each $s \in G$ and $f \in m(G)$, we define $r_{s} f$ in $m(G)$ given by

$$
\left(r_{s} f\right)(t)=f(t+s) \quad \forall t \in G .
$$

Let $X$ be a subspace of $m(G)$ and $\mu$ be an element of $X^{*}$ (the dual space of $X$ ). Then, we denote by $\mu(f)$ the value of $\mu$ at $f \in X$. To specify the variable $t$, we write the value $\mu(f)$ by $\mu(t)\langle f(t)\rangle$ or $\int f(t) d \mu(t)$. When $X$ contains a constant 1 , an element $\mu$ of $X^{*}$ is called a mean on $X$ if $\|\mu\|=\mu(1)=1$. Further, let $X$ be invariant under $r_{s}$ for all $s \in G$. Then, a mean $\mu$ on $X$ is said to be invariant if $\mu\left(r_{s} f\right)=\mu(f)$ for all $s \in G$ and $f \in X$. For $s \in G$, we can define a point evaluation $\delta_{s}$ by $\delta_{s}(f)=f(s)$ for every $f \in m(G)$. A convex combination of point evaluations is called a finite mean on $G$. Recently, the notion of the almost nonexpansive curve was introduced by Rouhani [5] and Kada and Takahashi [8]. 
Let $u(\cdot): G \mapsto H$ be a function, in what follows we refer to such $u(\cdot)$ as a curve in $H$. A bounded function $u$ is called an almost nonexpansive curve if there exists a function $\varepsilon: G \times G \rightarrow \mathbb{R}$ with $\lim _{s \cdot t \in G} \varepsilon(s, t)=0$, such that

$$
\|u(h+s)-u(h+t)\|^{2} \leq\|u(s)-u(t)\|^{2}+\varepsilon(s, t) \quad \forall s, t, h \in G .
$$

In the case $\varepsilon(s, t)=0$ for all $s, t \in G, u$ is called a nonexpansive curve.

Now, we define the concept of the asymptotically almost nonexpansive curve.

Definition 2.1. The curve $u(\cdot)$ is said to be asymptotically almost nonexpansive if the following conditions are satisfied:

(1) $\|u(h+t)-u(h+s)\|^{2} \leq\|u(t)-u(s)\|^{2}+\varepsilon(t, s, h)$ for all $t, s, h \in G$, where $\varepsilon(t, s, h) \geq 0$ for all $t, s, h \in G$;

(2) for an arbitrary $\varepsilon>0$ there exists $t_{0} \in G$, and for each $t \succcurlyeq t_{0}$ there exists $h_{t}=h(\varepsilon, t) \in G$ such that

$$
\varepsilon(t, s, h)<\varepsilon \quad \forall t \succcurlyeq t_{0}, s \succcurlyeq t_{0}, h \succcurlyeq h_{t} .
$$

Note that, if $u(\cdot)$ is bounded then condition (1) is equivalent to

$$
\|u(h+t)-u(h+s)\| \leq\|u(t)-u(s)\|+\varepsilon_{1}(t, s, h) \quad \forall t, s, h \in G,
$$

where $\varepsilon_{1}(t, s, h)$ satisfies the same condition (2) as $\varepsilon(t, s, h)$. We denote by $L(u)$ the following subset (possibly empty) of $H$ :

$$
L(u)=\left\{z \in H: \lim _{t \in G}\|u(t)-z\| \text { exists }\right\}
$$

Throughout the rest of this paper, $u(\cdot)$ is a bounded asymptotically almost nonexpansive curve and $X$ is a subspace of $m(G)$ containing constants invariant under $r_{s}$ for each $s \in G$. Furthermore, suppose that for each $x \in H$, the function $t \mapsto\|u(t)-x\|^{2}$ is in $X$. Then by Riesz theorem, there exists a unique element $u_{\mu}$ in $H$ such that

$$
\mu_{t}(u(t), x)=\left(u_{\mu}, x\right) \quad \forall x \in H .
$$

We denote $u_{\mu}$ by $\mu_{t}\langle u(t)\rangle$. If $\mu$ is a finite mean on $G$,

$$
\mu=\sum_{i=1}^{n} a_{i} \delta_{t_{i}} \quad\left(t_{i} \in G, a_{i} \geq 0,1 \leq i \leq n, \sum_{i=1}^{n} a_{i}=1\right),
$$

then

$$
\mu_{t}\langle u(t)\rangle=\sum_{i=1}^{n} a_{i} u\left(t_{i}\right) .
$$

We denote by $\omega_{w}(u)$ the set of all weak limits of subnets of the net $\{u(t): t \in G\}$. 


\section{Asymptotic behavior of curves}

We begin with the following lemmas and proposition which play an important role in the proof of our main theorems.

LEMMA 3.1. Let $u(\cdot)$ be a bounded asymptotically almost nonexpansive curve. Then the set $L(u)$ (possibly empty) is closed and convex.

Proof. We can show the closedness from this inequality,

$$
\begin{aligned}
& |\|u(t)-x\|-\|u(s)-x\|| \\
& \quad=\left|\|u(t)-x\|-\left\|u(t)-x_{n}\right\|+\left\|u(t)-x_{n}\right\|-\left\|u(s)-x_{n}\right\|+\left\|u(s)-x_{n}\right\|-\|u(s)-x\|\right| \\
& \quad \leq\left|\|u(t)-x\|-\left\|u(t)-x_{n}\right\|\right|+\left|\left\|u(t)-x_{n}\right\|-\left\|u(s)-x_{n}\right\|\right|+\left|\left\|u(s)-x_{n}\right\|-\|u(s)-x\|\right| \\
& \quad \leq 2\left\|x_{n}-x\right\|+\left|\left\|u(t)-x_{n}\right\|-\left\|u(s)-x_{n}\right\|\right| .
\end{aligned}
$$

And also, the convexity follows from the equality

$$
\begin{aligned}
\left\|u(t)-\left(\lambda q_{1}+(1-\lambda) q_{2}\right)\right\|^{2}= & \lambda\left\|u(t)-q_{1}\right\|^{2}+(1-\lambda)\left\|u(t)-q_{2}\right\|^{2} \\
& -\lambda(1-\lambda)\left\|q_{1}-q_{2}\right\|^{2} .
\end{aligned}
$$

Proposition 3.2. The set $\bigcap_{s \in G} \overline{\operatorname{conv}}\{u(t): t \succcurlyeq s\} \cap L(u)$ consists of at most one point.

Proof. Suppose that $L(u) \neq \emptyset$. Let $p$ be the unique asymptotic center of $\{u(t): t \in G\}$ in $L(u)$ and $x \in \bigcap_{s \in G} \overline{\operatorname{conv}}\{u(t): t \succcurlyeq s\} \cap L(u)$. We conclude the proof by showing that $x=p$. Since

$$
\|u(t)-x\|^{2}=\|u(t)-p\|^{2}+\|x-p\|^{2}+2(u(t)-p, p-x),
$$

we have

$$
2 \lim _{t \in G}(u(t)-p, p-x)+\|p-x\|^{2} \geq 0 .
$$

For any $\varepsilon>0$, there exists $t_{0} \in G$ such that

$$
2(u(t)-p, p-x)+\|p-x\|^{2} \geq-\varepsilon \quad \forall t \succcurlyeq t_{0} .
$$

Since $x \in \overline{\operatorname{conv}}\left\{u(t): t \succcurlyeq t_{0}\right\}$, it follows that

$$
2(x-p, p-x)+\|p-x\|^{2} \geq-\varepsilon,
$$

that is, $\|p-x\|^{2} \leq \varepsilon$. Since $\varepsilon>0$ is arbitrary, we have $x=p$. This completes the proof.

Since $G$ is commutative, there exists a net $\left\{\lambda_{\alpha}: \alpha \in I\right\}$ of finite means on $G$ such that

$$
\lim _{\alpha \in I}\left\|\lambda_{\alpha}-r_{s}^{*} \lambda_{\alpha}\right\|=0 \quad \forall s \in G
$$

where $I$ is a directed set and $r_{s}^{*}$ is the conjugate of $r_{s}$ (see [4]). 
Lemma 3.3. $\lambda_{\alpha}(t)\langle u(t+h)\rangle$ converges weakly to an element $p$ in $\bigcap_{s \in G} \overline{\operatorname{conv}}\{u(t): t \succcurlyeq s\}$ $\cap L(u)$ uniformly in $h \in G$.

Proof. For any $\left\{t_{\alpha}\right\} \in G$, let $W$ be the set of all weak limit points of $\lambda_{\alpha}(t)\left\langle u\left(t+t_{\alpha}\right)\right\rangle$. In view of Proposition 3.2, it suffices to show that

$$
W \subset \bigcap_{s \in G} \overline{\operatorname{conv}}\{u(t): t \succcurlyeq s\} \cap L(u) .
$$

To show this, let $\left\{t_{\alpha_{\beta}}: \beta \in J\right\}$ be a subnet of $\left\{t_{\alpha}: \alpha \in B\right\}$ such that $\lambda_{\alpha_{\beta}}(t)\left\langle u\left(t+t_{\alpha_{\beta}}\right)\right\rangle$ converges weakly to some $z$ in $H$, where $J$ is a directed set. For any $\varepsilon>0$, there exists $t_{\varepsilon} \in G$ such that for any $t \succcurlyeq t_{\epsilon}$, there exists $h_{t} \in G$ such that

$$
\varepsilon(t, s, h)<\varepsilon \quad \forall t, s \succcurlyeq t_{\epsilon}, h \succcurlyeq h_{t} .
$$

Then

$$
\begin{aligned}
\| u(h+ & t)-z\left\|^{2}-\right\| u(t)-z \|^{2}-2\left(u(h+t)-u(t), \lambda_{\alpha_{\beta}}(s)\left\langle u\left(s+t_{\alpha_{\beta}}+t_{\varepsilon}\right)\right\rangle-z\right) \\
= & \left(u(h+t)-u(t), u(h+t)+u(t)-2 \lambda_{\alpha_{\beta}}(s)\left\langle u\left(s+t_{\alpha_{\beta}}+t_{\varepsilon}\right)\right\rangle\right) \\
= & \lambda_{\alpha_{\beta}}(s)\left(\left\|u(h+t)-u\left(s+t_{\alpha_{\beta}}+t_{\varepsilon}\right)\right\|^{2}-\left\|u(t)-u\left(s+t_{\alpha_{\beta}}+t_{\varepsilon}\right)\right\|^{2}\right) \\
\leq & \lambda_{\alpha_{\beta}}(s)\left(\left\|u(h+t)-u\left(h+s+t_{\alpha_{\beta}}+t_{\varepsilon}\right)\right\|^{2}-\left\|u(t)-u\left(s+t_{\alpha_{\beta}}+t_{\varepsilon}\right)\right\|^{2}\right) \\
& +4 M^{2}\left\|\lambda_{\alpha_{\beta}}-r_{h}^{*} \lambda_{\alpha_{\beta}}\right\| \\
< & +4 M^{2}\left\|\lambda_{\alpha_{\beta}}-l_{h}^{*} \lambda_{\alpha_{\beta}}\right\|
\end{aligned}
$$

for all $t \succcurlyeq t_{\epsilon}$ and $h \succcurlyeq h_{t}$, where $M=\sup _{t \in G}\|u(t)\|$. Note that $\lambda_{\alpha_{\beta}}(t)\left\langle u\left(t+t_{\alpha_{\beta}}+t_{\varepsilon}\right)\right\rangle$ converges weakly to $z$. For fixed $t \succcurlyeq t_{\varepsilon}$ and $h \succcurlyeq h_{t}$, taking the limit for $\beta \in J$, we have

$$
\|u(h+t)-z\|^{2}-\|u(t)-z\|^{2} \leq \varepsilon \quad \forall t \succcurlyeq t_{\varepsilon}, h \succcurlyeq h_{t} .
$$

Therefore,

$$
\inf _{s \in G \tau \succcurlyeq s} \sup _{\tau}\|u(\tau)-z\|^{2} \leq\|u(t)-z\|^{2}+\varepsilon \quad \forall t \succcurlyeq t_{\varepsilon}
$$

and hence

$$
\inf _{s \in G} \sup _{\tau \succcurlyeq s}\|u(\tau)-z\|^{2} \leq \sup _{s \in G} \inf _{\tau \succcurlyeq s}\|u(\tau)-z\|^{2}+\varepsilon .
$$

Since $\varepsilon>0$ is arbitrary, we have $z \in L(u)$.

Now, we show that $z \in \bigcap_{s \in G} \overline{\operatorname{conv}}\{u(t): t \succcurlyeq s\}$. For each $s \in G$, since $\lambda_{\alpha_{\beta}}(t)\langle u(t+$ $\left.\left.t_{\alpha_{\beta}}+s\right)\right\rangle \in \overline{\operatorname{conv}}\{u(t): t \succcurlyeq s\}$, we get $z \in \bigcap_{s \in G} \overline{\operatorname{conv}}\{u(t): t \succcurlyeq s\}$. This completes the proof.

Now, we can prove the ergodic convergence theorem for asymptotically almost nonexpansive curves.

A net $\left\{\mu_{\alpha}: \alpha \in A\right\}$ of continuous linear functionals on $X$ is called strongly regular if it satisfies the following conditions: 
152 Nonlinear ergodic theorems

(a) $\sup _{\alpha \in A}\left\|\mu_{\alpha}\right\|<+\infty$;

(b) $\lim _{\alpha \in A} \mu_{\alpha}(1)=1$;

(c) $\lim _{\alpha \in A}\left\|\mu_{\alpha}-r_{s}^{*} \mu_{\alpha}\right\|=0$ for every $s \in G$.

THEOREM 3.4. Let $\left\{\mu_{\alpha}: \alpha \in A\right\}$ be a strongly regular net of continuous linear functional on $X$. Then there exists $p \in \bigcap_{s \in G} \overline{\operatorname{conv}}\{u(t): t \succcurlyeq s\} \cap L(u)$ such that

$$
w-\lim _{\alpha \in A} \int u(t+h) d \mu_{\alpha}(t)=p \quad \text { uniformly in } h \in G .
$$

Moreover, $u_{\mu}=p$ for each invariant mean $\mu$.

Proof. By Lemma 3.3, there exists $p \in \bigcap_{s \in G} \overline{\operatorname{conv}}\{u(t): t \succcurlyeq s\} \bigcap L(u)$ and for any $\varepsilon>0$ and $y_{0} \in H$ with $\left\|y_{0}\right\|=1$, there exists $\alpha_{0} \in B$ such that

$$
\left|\left(\lambda_{\alpha_{0}}(t)\langle u(t+h)\rangle-p, y_{0}\right)\right|<\frac{\varepsilon}{\sup _{\alpha \in A}\left\|\mu_{\alpha}\right\|} \quad \forall h \in G .
$$

Suppose that

$$
\lambda_{\alpha_{0}}=\sum_{i=1}^{n} a_{i} \delta_{t_{i}}, \quad t_{i} \in G, a_{i} \geq 0, i=1,2, \ldots, n, \sum_{i=1}^{n} a_{i}=1 .
$$

Since $\left\{\mu_{\alpha}: \alpha \in A\right\}$ is strongly regular, there exists $\alpha_{1} \in A$ such that

$$
\begin{gathered}
\left|\mu_{\alpha}(1)-1\right|<\frac{\varepsilon}{(\|p\|+1)}, \\
\left\|\mu_{\alpha}-r_{s_{i}}^{*} \mu_{\alpha}\right\|<\frac{\varepsilon}{M}, \quad 1 \leq i \leq n, \forall \alpha \succcurlyeq \alpha_{1},
\end{gathered}
$$

where $M=\sup \{\|u(t)\|: t \in G\}$. Since for all $\alpha \succcurlyeq \alpha_{1}, h \in G$,

$$
\begin{aligned}
& \left|\left(\int \lambda_{\alpha_{0}}(t)\langle u(t+s+h)\rangle d \mu_{\alpha}(s)-p, y_{0}\right)\right| \\
& =\left|\int\left(\lambda_{\alpha_{0}}(t)\langle u(t+s+h)\rangle-p, y_{0}\right) d \mu_{\alpha}(s)-\left(p, y_{0}\right)\left(\mu_{\alpha}(1)-1\right)\right| \\
& \quad \leq \sup _{\alpha \in A}\left\|\mu_{\alpha}\right\| \sup _{s \in G}\left|\left(\lambda_{\alpha_{0}}(t)\langle u(t+s+h)\rangle-p, y_{0}\right)\right|+\varepsilon \leq 2 \varepsilon, \\
& \left|\left(\int u(s+h) d \mu_{\alpha}(s), y_{0}\right)-\left(\int \lambda_{\alpha_{0}}(t)\langle u(t+s+h)\rangle d \mu_{\alpha}(s), y_{0}\right)\right| \\
& \quad=\left|\left(\int\left(u(s+h)-\sum_{i=1}^{n} a_{i} u\left(t_{i}+s+h\right)\right) d \mu_{\alpha}(s), y_{0}\right)\right| \\
& \quad \leq \sum_{i=1}^{n} a_{i} M\left\|\mu_{\alpha}-r_{t_{i}}^{*} \mu_{\alpha}\right\|<\varepsilon .
\end{aligned}
$$

Thus, we obtain, for all $\alpha \succcurlyeq \alpha_{1}, h \in G$,

$$
\left|\left(\int u(s+h) d \mu_{\alpha}(s)-p, y_{0}\right)\right|<3 \varepsilon .
$$


This completes the proof.

THEOREM 3.5. Let $u(\cdot)$ be a bounded asymptotically almost nonexpansive curve. Then the following conditions are equivalent:

(1) $w-\lim _{t \in G} u(t)$ exists;

(2) $\omega_{w}(u) \subset L(u)$;

(3) $w-\lim _{t \in G}(u(h+t)-u(t))=0$ for every $h \in G$.

Proof. (3) $\Rightarrow(2)$. Let $\varepsilon>0$. Then there exists $t_{\varepsilon} \in G$ and for each $t \succcurlyeq t_{\varepsilon}$ there exists $h_{t} \in G$ such that

$$
\varepsilon(t, s, h)<\varepsilon \quad \forall t, s \succcurlyeq t_{\varepsilon}, h \succcurlyeq h_{t} .
$$

Let $z \in \omega_{w}(u)$. Then we can take a subnet $\left\{u\left(t_{\alpha}\right): \alpha \in J\right\}$ with $t_{\alpha} \succcurlyeq t_{\varepsilon}$ for each $\alpha \in J$ and

$$
w-\lim _{\alpha \in J} u\left(t_{\alpha}\right)=z
$$

Since $L(u)$ is nonempty by Lemma 3.3, let $p \in L(u)$. Since for each $t \succcurlyeq t_{\varepsilon}$ and $h \succcurlyeq h_{t}$,

$$
\begin{aligned}
\left\|u\left(h+t_{\alpha}\right)-p\right\|^{2}- & \left\|u\left(t_{\alpha}\right)-p\right\|^{2}+2\left(u\left(h+t_{\alpha}\right)-u\left(t_{\alpha}\right), p-z\right) \\
= & \left\|u\left(h+t_{\alpha}\right)-z\right\|^{2}-\left\|u\left(t_{\alpha}\right)-z\right\|^{2} \\
= & \left\|u\left(h+t_{\alpha}\right)-u(h+t)\right\|^{2}+\|u(h+t)-z\|^{2} \\
& +2\left(u\left(h+t_{\alpha}\right)-u(h+t), u(h+t)-z\right)-\left\|u\left(t_{\alpha}\right)-z\right\|^{2} \\
\leq & \left\|u\left(t_{\alpha}\right)-u(t)\right\|^{2}+\varepsilon+\|u(h+t)-z\|^{2} \\
& +2\left(u\left(h+t_{\alpha}\right)-u(h+t), u(h+t)-z\right)-\left\|u\left(t_{\alpha}\right)-z\right\|^{2} \\
= & \|u(h+t)-z\|^{2}+\|u(t)-z\|^{2}+2\left(u\left(t_{\alpha}\right)-z, z-u(t)\right) \\
& +2\left(u\left(h+t_{\alpha}\right)-u(h+t), u(h+t)-z\right)+\varepsilon,
\end{aligned}
$$

for fixed $t \succcurlyeq t_{\varepsilon}$ and $h \succcurlyeq h_{t}$. Taking the limit for $\alpha \in J$, we have

$$
\|u(h+t)-z\|^{2} \leq\|u(t)-z\|^{2}+\varepsilon .
$$

This implies $z \in L(u)$ in the same way as in Lemma 3.3.

$(2) \Rightarrow(1)$. Since $\omega_{w}(u) \subset \bigcap_{s \in G} \overline{\operatorname{conv}}\{u(t): t \geq s\}, \omega_{w}(u)$ is a singleton from Proposition 3.2. This implies (1) holds.

$(1) \Rightarrow(3)$. It is clear.

\section{Asymptotic behavior of almost-orbits}

In this section, using the main results in Section 3, we prove the ergodic theorems and weak convergence theorems for almost-orbits of commutative semigroups of asymptotically nonexpansive type mappings with nonconvex domains. 
154 Nonlinear ergodic theorems

Let $C$ be a nonempty subset of a Hilbert space $H$ and $\mathfrak{s}=\{T(t): t \in G\}$ be a family of mappings from $C$ into itself. Recall that $\Im$ is said to be a commutative semigroup of asymptotically nonexpansive type mappings on $C$ if the following conditions are satisfied:

(a) $T(t+s) x=T(t) T(s) x$ for all $t, s \in G$ and $x \in C$;

(b) for each $x \in C$ and $t \in G$, there exists $\alpha(t, x) \geq 0$ such that

$$
\|T(t) x-T(t) y\| \leq\|x-y\|+\alpha(t, x) \quad \forall y \in C,
$$

with

$$
\lim _{t \in G} \alpha(t, x)=0 \quad \forall x \in C .
$$

A function $u(\cdot): G \mapsto C$ is said to be an almost-orbit of $\Im=\{T(t): t \in G\}$ if

$$
\lim _{t \in G}\left[\sup _{h \in G}\|u(h+t)-T(h) u(t)\|\right]=0 .
$$

Throughout the rest of this section, $\mathfrak{s}=\{T(t): t \in G\}$ is a commutative semigroup of asymptotically nonexpansive type mappings on $C, u(\cdot): G \mapsto C$ is a bounded almost-orbit of $\mathfrak{I}=\{T(t): t \in G\}$, and $X$ is a subspace of $m(G)$ containing constants invariant under $r_{s}$ for each $s \in G$. Furthermore, suppose that for each $x \in H$, the function $t \mapsto\|u(t)-x\|^{2}$ is in $X$. Denote by $F(\Im)$ the set of common fixed points of $\mathfrak{s}=\{T(t): t \in G\}$.

We begin with the following lemmas.

Lemma 4.1. Let $u(\cdot)$ be a bounded almost-orbit of the commutative semigroup $\mathfrak{s}=$ $\{T(t): t \in G\}$ of asymptotically nonexpansive type mappings on $C$. Then it is an asymptotically almost nonexpansive curve.

Proof. Put $\varphi(t)=\sup _{h \in G}\|u(h+t)-T(h) u(t)\|$. Then $\lim _{t \in G} \varphi(t)=0$. Since

$$
\begin{aligned}
\|u(h+t)-u(h+s)\| \leq & \|u(h+t)-T(h) u(t)\|+\|T(h) u(t)-T(h) u(s)\| \\
& +\|u(h+s)-T(h) u(s)\| \\
\leq & \varphi(t)+\varphi(s)+\alpha(h, u(t))+\|u(t)-u(s)\|,
\end{aligned}
$$

for every $h, t, s \in G$. It is easily seen that $u(\cdot)$ is an asymptotically almost nonexpansive curve.

Lemma 4.2. If $u(\cdot)$ and $v(\cdot)$ are almost-orbits of $\mathfrak{\Im}$, then $\lim _{t \in G}\|u(t)-v(t)\|$ exists. Furthermore, we have $F(\mathfrak{\Im}) \subseteq L(u)$.

Proof. Set

$$
\begin{aligned}
& \varphi(t)=\sup _{s \in G}\|u(s+t)-T(s) u(t)\|, \\
& \psi(t)=\sup _{s \in G}\|v(s+t)-T(s) v(t)\| .
\end{aligned}
$$


Then, $\lim _{t \in G} \varphi(t)=\lim _{t \in G} \psi(t)=0$. Since for each $t, s \in G$,

$$
\begin{aligned}
\|u(s+t)-v(s+t)\| \leq & \|u(s+t)-T(s) u(t)\|+\|T(s) u(t)-T(s) v(t)\| \\
& +\|v(s+t)-T(s) v(t)\| \\
\leq & \varphi(t)+\psi(t)+\alpha(s, u(t))+\|u(t)-v(t)\|, \\
\inf \sup _{s \in G \tau s}\|u(\tau)-v(\tau)\| \leq & \varphi(t)+\psi(t)+\|u(t)-v(t)\| .
\end{aligned}
$$

It follows that

$$
\inf _{s \in G} \sup _{\tau \succcurlyeq s}\|u(\tau)-v(\tau)\| \leq \sup _{s \in G} \inf _{\tau \succcurlyeq s}\|u(\tau)-v(\tau)\|,
$$

which complete the proof of the first part. The second part is obvious.

We can prove the following proposition from Lemma 4.2 and Proposition 3.2.

Proposition 4.3. The set $\bigcap_{s \in G} \overline{\operatorname{conv}}\{u(t): t \succcurlyeq s\} \cap F(\Im)$ consists of at most one point.

From Theorem 3.4, we can prove the following theorem which is an extension of the result of Tan and $\mathrm{Xu}$ [17] in many directions.

THeOrem 4.4. Let $C$ be a nonempty subset of $H, \mathfrak{s}=\{T(t): t \in G\}$ a commutative semigroup of asymptotically nonexpansive type mappings on $C$, and $u(\cdot)$ be a bounded almost-orbit of $\Im$. If $\left\{\mu_{\alpha}: \alpha \in A\right\}$ is a strongly regular net of continuous linear functional on $X$, then

$$
w-\lim _{\alpha \in A} \int u(t+h) d \mu_{\alpha}(t)=p \in \bigcap_{s \in G} \overline{\operatorname{conv}}\{u(t): t \succcurlyeq s\} \bigcap L(u)
$$

uniformly in $h \in G$. Further, if each $T(t)$ is continuous and $\bigcap_{s \in G} \overline{\operatorname{conv}}\{u(t): t \succcurlyeq s\} \subset$ $C$, then $p \in F(\Im)$.

Proof. By Lemma 4.1 and Theorem 3.4, we need only to prove that if each $T(t)$ is continuous and $\bigcap_{s \in G} \overline{\operatorname{conv}}\{u(t): t \succcurlyeq s\} \subset C$, then $p \in F(\Im)$. By assumption, we have $p \in C$. Let $0<\varepsilon \leq 1$. Then there exists $t_{1} \in G$ such that

$$
\begin{gathered}
\varphi(t)=\sup _{h \in G}\|T(h) u(t)-u(h+t)\|<\frac{\varepsilon}{8 d}, \\
\alpha(t, p)<\frac{\varepsilon}{8 d},
\end{gathered}
$$

for each $t \succcurlyeq t_{1}$, where $d=1+\sup \{\|u(t)-p\|: t \in G\}$. Since

$$
\begin{aligned}
\| T(s) & p-p\left\|^{2}+2\left(u\left(s+t+t_{1}\right)-p, p-T(s) p\right)+\right\| u\left(s+t+t_{1}\right)-p \|^{2} \\
= & \left\|u\left(s+t+t_{1}\right)-T(s) u\left(t+t_{1}\right)\right\|^{2}+\left\|T(s) u\left(t+t_{1}\right)-T(s) p\right\|^{2} \\
& +2\left(u\left(s+t+t_{1}\right)-T(s) u\left(t+t_{1}\right), T(s) u\left(t+t_{1}\right)-T(s) p\right) \\
\leq & \left\|u\left(t+t_{1}\right)-p\right\|^{2}+\varepsilon
\end{aligned}
$$


156 Nonlinear ergodic theorems

for $s \succcurlyeq t_{1}$, this implies that

$$
\begin{gathered}
\|T(s) p-p\|^{2} \mu_{\alpha}(1)+2 \mu_{\alpha}(t)\left(u\left(s+t+t_{1}\right)-p, p-T(s) p\right) \\
\leq\left(\mu_{\alpha}-r_{s}^{*} \mu_{\alpha}\right)(t)\left\|u\left(t+t_{1}\right)-p\right\|^{2}+\mu_{\alpha}(1) \varepsilon .
\end{gathered}
$$

Taking the limsup for $\alpha \in A$, we get

$$
\|T(s) p-p\|^{2} \leq \varepsilon \quad \forall s \succcurlyeq t_{1}
$$

It follows that $T(t) p$ is convergent strongly to $p$, therefore, $p \in F(\Im)$ by the continuity of $\{T(t): t \in G\}$. This completes the proof.

Let $\mathrm{AO}(\mathfrak{I})$ be the set of all almost-orbits of $\mathfrak{I}$. Then for each $h \in G$ and $u \in \mathrm{AO}(\mathfrak{I})$, the function $v: G \mapsto C$, defined by $v(t)=T(h) u(t)$, is also an almost-orbit of $\Im$. In fact, as before, we set $\varphi(t)=\sup _{s \in G}\|u(s+t)-T(s) u(t)\|$. Since

$$
\begin{aligned}
\|v(s+t)-T(s) v(t)\|= & \|T(h) u(s+t)-T(s) T(h) u(t)\| \\
\leq & \|T(h) u(s+t)-u(h+s+t)\| \\
& +\|u(h+s+t)-T(s+h) u(t)\| \\
\leq & \varphi(s+t)+\varphi(t),
\end{aligned}
$$

the result follows.

Using Theorem 4.4, we have the following ergodic retraction theorem.

THEOREM 4.5. Let $C$ be a nonempty bounded subset of a Hilbert space $H$ and let $\Im$ be a commutative semigroup of asymptotically nonexpansive type mappings on $C$ such that each $T(t)$ is continuous. Then for an invariant mean $\mu$, the mapping $P: u \mapsto u_{\mu}$ is a unique retraction from the set $\mathrm{AO}(\mathfrak{\Im})$ onto $F(\Im)$ such that

(1) $P$ is nonexpansive in the sense that

$$
\|P u-P v\| \leq \lim _{t \in G}\|u(t)-v(t)\|
$$

(2) $P T(h) u=T(h) P u=P u$ for $u \in \mathrm{AO}(\mathfrak{I})$ and $h \in G$;

(3) $P u \in \bigcap_{s \in G} \overline{\operatorname{conv}}\{u(t): t \succcurlyeq s\}$ for $u \in \mathrm{AO}(\Im)$.

As a direct consequence of Theorem 3.5, we can prove the following theorem which is an extension of the Takahashi and Zhang [16]. Note that we do not assume $F(\Im)$ to be nonempty.

Theorem 4.6. Let $C$ be a nonempty subset of a Hilbert space $H$ and let $\mathfrak{s}$ be a commutative semigroup of asymptotically nonexpansive type mappings on $C$, and let 
$u(\cdot)$ be a bounded almost-orbit of $\mathfrak{\Im}$. Then $w-\lim _{t \in G} u(t)$ exists (in $\left.L(u)\right)$ if and only if $w-\lim _{t \in G}(u(h+t)-u(t))=0$ for all $h \in G$.

\section{Acknowledgement}

This work was supported by Korea Research Foundation Grant (KRF-99-041-D00025).

\section{References}

[1] J.-B. Baillon, Un théorème de type ergodique pour les contractions non linéaires dans un espace de Hilbert, C. R. Acad. Sci. Paris Sér. A-B 280 (1975), no. 22, 1511-1514. MR 51\#11205. Zbl 307.47006.

[2] J. B. Baillon and $\mathrm{H}$. Brezis, Une remarque sur le comportement asymptotique des semigroupes non linéaires, Houston J. Math. 2 (1976), no. 1, 5-7. MR 52\#15131. Zbl 318.47039.

[3] H. Brézis and F. E. Browder, Remarks on nonlinear ergodic theory, Advances in Math. 25 (1977), no. 2, 165-177. MR 57\#1218. Zbl 399.47058.

[4] M. M. Day, Amenable semigroups, Illinois J. Math. 1 (1957), 509-544. MR 19,1067c. Zbl 078.29402.

[5] B. Djafari Rouhani, Asymptotic behaviour of quasi-autonomous dissipative systems in Hilbert spaces, J. Math. Anal. Appl. 147 (1990), no. 2, 465-476. MR 91h:47069. Zbl 726.47032.

[6] N. Hirano and W. Takahashi, Nonlinear ergodic theorems for uniformaly lipschitzian semigroups in Hilbert spaces, J. Math. Anal. Appl. 127 (1987), 206-210.

[7] H. Ishihara and W. Takahashi, A nonlinear ergodic theorem for a reversible semigroup of Lipschitzian mappings in a Hilbert space, Proc. Amer. Math. Soc. 104 (1988), no. 2, 431-436. MR 90g:47120. Zbl 692.47010.

[8] O. Kada and W. Takahashi, Nonlinear ergodic theorems for almost nonexpansive curves over commutative semigroups, Topol. Methods Nonlinear Anal. 5 (1995), no. 2, 305324. MR 97f:47053. Zbl 876.47047.

[9] W. A. Kirk and R. Torrejón, Asymptotically nonexpansive semigroups in Banach spaces, Nonlinear Anal. 3 (1979), no. 1, 111-121 (1978). MR 82a:47062. Zbl 411.47035.

[10] A. T. M. Lau, K. Nishiura, and W. Takahashi, Nonlinear ergodic theorems for semigroups of nonexpansive mappings and left ideals, Nonlinear Anal. 26 (1996), no. 8, 1411-1427. MR 97b:47074. Zbl 880.47048.

[11] G. Li and J. Ma, Nonlinear ergodic theorem for semitopological semigroups of nonLipschitzian mappings in Banach spaces, Chinese Sci. Bull. 42 (1997), no. 1, 8-11. MR 98e:47110. Zbl 904.47063.

[12] G. Li and J. P. Ma, The asymptotic behavior of an asymptotically almost nonexpansive curve and an ergodic theorem, Chinese Ann. Math. Ser. A 17 (1996), no. 6, 729-736 (Chinese). MR 98c:47066.

[13] N. Mizoguchi and W. Takahashi, On the existence of fixed points and nonlinear ergodic retractions for Lipschitzian semigroups in Hilbert spaces, Nonlinear Anal. 13 (1990), 69-97.

[14] W. Takahashi, A nonlinear ergodic theorem for a reversible semigroup of nonexpansive mappings in a Hilbert space, Proc. Amer. Math. Soc. 97 (1986), no. 1, 55-58. MR 88f:47051.

[15] _ Fixed point theorem and nonlinear ergodic theorem for nonexpansive semigroups without convexity, Canad. J. Math. 44 (1992), no. 4, 880-887. MR 93j:47091. Zbl 786.47047. 
158 Nonlinear ergodic theorems

[16] W. Takahashi and P. J. Zhang, Asymptotic behavior of almost-orbits of reversible semigroups of Lipschitzian mappings, J. Math. Anal. Appl. 142 (1989), no. 1, 242-249. MR 90g:47121. Zbl 695.47062.

[17] K. K. Tan and H. K. Xu, Asymptotic behavior of almost-orbits of nonlinear semigroups of non-Lipschitzian mappings in Hilbert spaces, Proc. Amer. Math. Soc. 117 (1993), no. 2, 385-393. MR 93d:47128. Zbl 807.47055.

Gang Li: Department of Mathematics, Yangzhou University, Yangzhou 225002, CHINA

E-mail address: yzlgang@pub.yz.jsinfo.net

Jong Kyu Kim: Department of Mathematics, Kyungnam University, Masan, Kyungnam 631-701, KoREA

E-mail address: jongkyuk@ kyungnam.ac.kr 


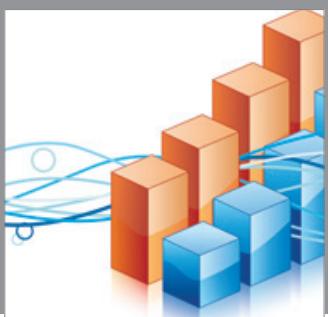

Advances in

Operations Research

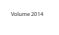

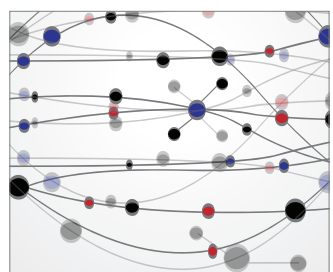

\section{The Scientific} World Journal
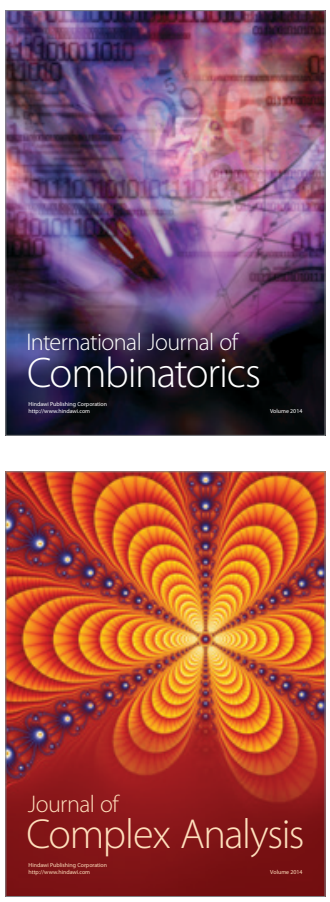

International Journal of

Mathematics and

Mathematical

Sciences
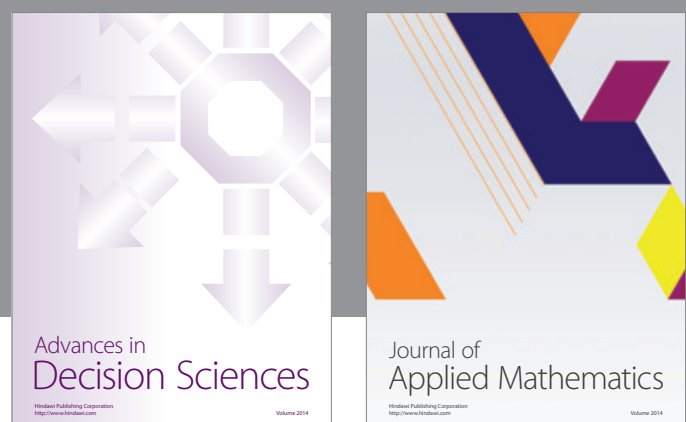

Journal of

Applied Mathematics
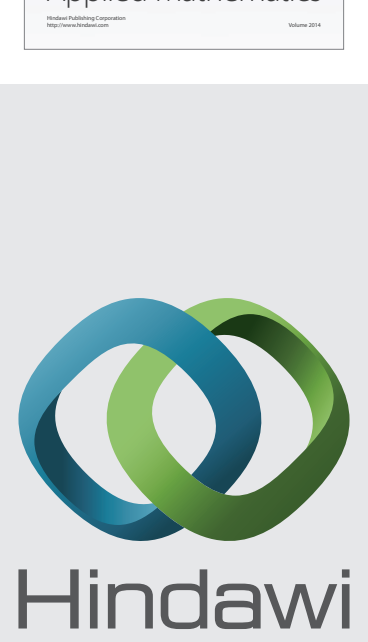

Submit your manuscripts at http://www.hindawi.com
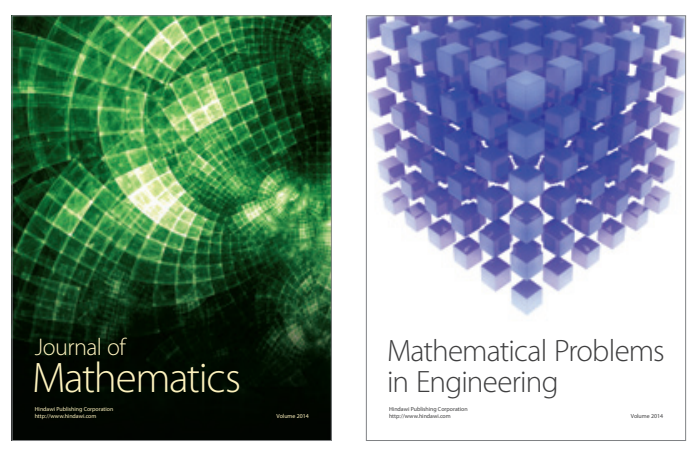

Mathematical Problems in Engineering
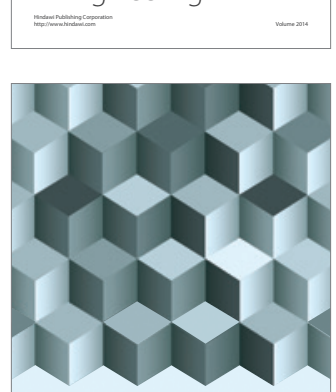

Journal of

Function Spaces
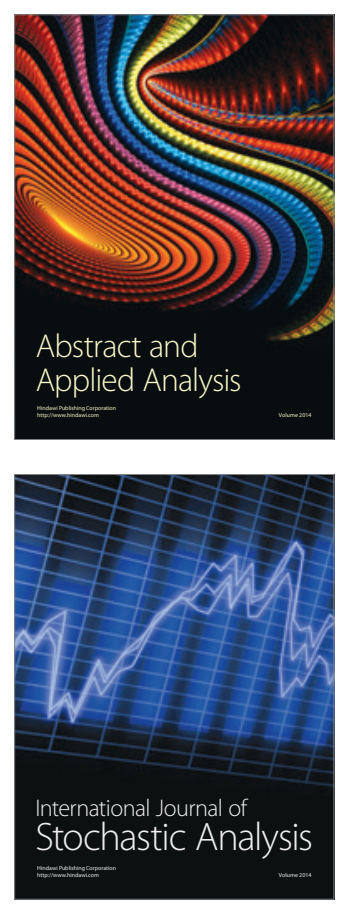

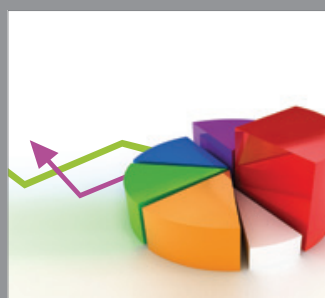

ournal of

Probability and Statistics

Promensencen
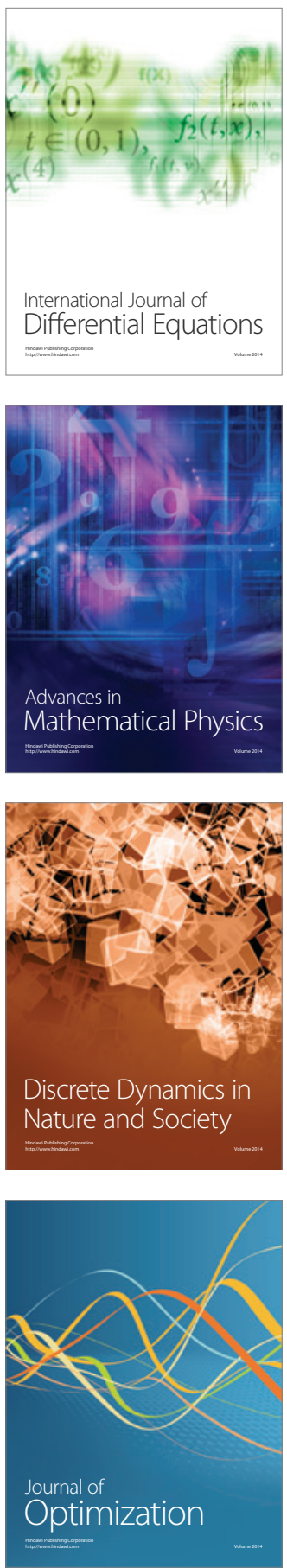